\title{
3D Carto-Graphics - Principles, Methods and Examples for Interactive Atlases
}

\author{
René Sieber*, Remo Eichenberger, Lorenz Hurni \\ Institute of Cartography and Geoinformation,ETH Zurich, \{sieberr|remoe|lhurni\}@ethz.ch \\ * Corresponding author
}

Keywords: Atlas Concepts, 3D Cartography, 3D Map Design, Cartographic Representations

\begin{abstract}
:
Atlases are designed to visualize, explore and analyze topographic and thematic information in a geographic environment. As 3D data and real-time display techniques are increasingly available, a trend towards 3D atlases can be observed like the newly released Earth 3D Amazing Atlas (2017) and the Atlas of Switzerland - online (2016). While creating such interactive 3D atlases, editors are often confronted with the question: How realistic should a cartographic 3D representation look like? Can we introduce some visualization guidelines or even rules to determine the ,graphic style" of cartographic 3D elements? 3D visualizations tend to let users ask for more and more details, leading to photorealistic representations. But photorealism is mostly not suited to pin point the characteristics of a theme; obviously, a creek or a trail would hardly be recognized in a forest area. As Goralski $(2009$, p.3) states: "3D maps are not meant to be realistic 3D representations of the real world. As in other map types, cartographic rules of abstraction, symbolization and generalization have to be used, to assure efficient transfer of the depicted geographical information, tailored to the purpose, and suitable for the target map user."
\end{abstract}

In our presentation, we will clarify the term of 3D carto-graphics, depict principles, and describe suitable methods and corresponding techniques. In the context of the national Atlas of Switzerland, we will apply and examine these design concepts for 3D representations within the 3D mapping space (Sieber et al. 2013).

A carto-graphic style for 3D is based on 2D cartographic rules (Imhof 1965) and non-photorealistic computer graphics (Doellner 2012, Bodum 2005). Principles concerning 3D modeling are fundamental for the different representational aspects. In this context, we will discuss principles such as a degree of realism, the level of visual complexity of 3D maps, the graphic quality of map elements, the 3D visualization and symbolization (Near-Far/Distance-Density problem), etc. considering dynamic and real-time applications. As an example of a 3D principle, the visualization should always originate from 3D data; thus a 2D map is a special case of a 3D map (Sieber et al. 2012).

Methods and techniques of 3D modeling affect the whole 3D scene consisting of terrain/topography, and different map objects. We will present some ideas and techniques how to treat 3D topography, and objects like point symbols, charts, lines, areas and solid objects considering real-time interaction. As an example of such methods recommended in the field of 3D topography, DTMs should be based on high-resolution and smoothed TINs applying techniques of low poly height fields (Ferguson 2013). Adaptive DTM smoothing using topographic position index (TPI) and filtering techniques are also taken into consideration (Guisan et al. 1999, Kettunen et al. 2017). For appropriate relief shading, an exemplary approach using smoothing and enhance techniques is suggested (Geisthövel 2017).

To illustrate the described methods and techniques, we present and discuss characteristic examples from various application fields. Examples may come from cartography, computer graphics, and even from data journalism and infographics. In order to demonstrate the feasibility and the usability of this approach, we plan to implement a set of 3D visualizations, which can be interacted with in real-time by means of the Virtual Globe engine of the Atlas of Switzerland - online. 